\title{
PREVALÊNCIA DA CISTICERCOSE EM CARCAÇAS DE BOVINOS ABATIDOS EM MATADOUROS-FRIGORÍFICOS DO ESTADO DO RIO DE JANEIRO, SUBMETIDOS AO CONTROLE DO SERVIÇO DE INSPEÇÃO FEDERAL (SIF-RJ), NO PERÍODO DE 1997 A 2003*
}

\begin{abstract}
M.A.V. da C. Pereira' ${ }^{1}$ V.S. Schwanz ${ }^{1}$, C.G . Barbosa ${ }^{2}$
${ }^{1}$ UniversidadeEstadual doN orteFluminense, Centro deCiênciaseTecnol ogias Agropecuárias, Laboratório de Sanidade A nimal, Av. Alberto Lamego, 2000, CEP 28013-600, Campos dos Goytacazes, RJ, Brasil. E-mail: angel@uenf.br
\end{abstract}

RESUMO

A Cisticercose é uma doença parasitária, considerada zoonose, provocada pela presença das formas larvárias intermediárias das Taenia saginata Goeze, 1782 (=Cysticercus bovis) e Taenia sol ium Lineu, 1758 (=Cysticercus cellulosae), nostecidosbovinos, suínosou dohomem. A Organização M undial daSaúdeestimaquecercade 50.000.000 depessoas possuem Cisticercose, eque50.000 morremacada ano. O homeméoúnico hospedeiro definitivo daforma adultatanto daT saginata quanto daT solium, asadquirindo pelaingestão das carnessuínaou bovina, mal cozidas, águaealimentoscontaminados. O bovino eosuíno sãooshospedeirosintermediáriosqueadquiremaCisticercoseingerindo osovos provenientes dasfezes humanasinfectadas, quecontaminam pastos, verduras, legumeseprincipalmenteaágua. A ocorrênciadestamanifestação dínicaestádiretamenterelacionadacoma recariedade dascondições sanitáriaseo baixonível sócio-econômico-cultural da população. Sobo ponto devista dasaúdepública, oserviçodeinspeçãosanitáriadecarnes, éumacondiçãofundamental paraaproteção da população frenteao complexo teníase-cisticercoseeuma importantefontedemonitoramento da incidênciadecisticercoseemanimais. O impactodaocorrênciadacisticercoseestárelacionadoaperdas econômicas associadas à produção de alimentos, sendo esta perda da ordem de U $\$ 420.000 .000,00$ anuais na A mérica do Sul (OPAS, 1994). No Brasil, apesar do conhecimento de vários estados com elevada freqüência da doença, não houveatéo momento um estudo detal hado da zoonoseem toda federação. Em bovinos, acisticercoseéazoonoseparasitáriamaisfreqüentementediagnosticadaem matadouros frigoríficos, sendo a principal causa de condenações, seqüestros e aproveitamentos condicionais decarcaças. Estetrabal hotem como objetivo determinar aprevalênciadacisticercoseem carcaças debovinos abatidos em matadouros-frigoríficos, sob inspeção federal, no estado do Rio de Janeiro, no período de 1999 a 2003.

PALAVRAS-CHAVE: Cisticercose bovina, Rio de Janeiro, matadouro-frigorífico.

\section{ABSTRACT}

PREVALENCE OF CISTICERCOSIS IN SLAUGHTERED BOVINE CARCASSES IN SLAUGHTERHOUSES AND COLD STORES IN RIO DE JANEIRO STATE BRAZIL, SUBMITTED UNDER THE FEDERAL SURVEILLAN CE SERVICE (SIF-RJ) FROM 1997TO 2003. Cysticercosis is a parasitic disease considered as a zoonosis, caused by the presence of intermediate larvae from Taenia saginata Goeze, 1782 (=Cysticer cusbovis) and TaeniasoliumLineu, 1758 (=Cysticercuscellulosae) on bovine, swineand human tissues. TheWorld Health Organization has evaluated that around $50,000,000$ people are carriers and 50,000 die, worldwide, every year. Human being has been the single definitive host of the adult phase for both T. saginata and T. solium, by the consumption of uncooked bovineand swinemeat, water and contaminated food. Bovineand swinehavebeen the intermediatehosts of theseparasites, ingesting theeggsfrom infected human faeces contaminating

UniversidadeFederal Rural do Rio deJanei ro, Departamento de Matemática, Instituto deCiências Exatas, Seropédica, RJ, Brasil.

*TrabalhoéorigináriodeumProjetointitulado: "DiagnósticoeControledeZoonosesnaCominudadeMatadouro, Campos dos Goytacazes-RJ" com apoio da Fundação Carlos Chagas de A poio à Pesquisa no Estado do Rio de Janeiro (A PQ1FAPERJ-projeto individual). 
pastures, vegetables, legumes and mainly fresh water. Theoccurrenceof this disease has directly been related to the poor sanitary conditionsand thelow social-economicand cultural level of the human population. Under thepoint of view of Public H ealth themeat sanitary inspection service has been a fundamental condition for protecting people in regard to the teniasis-cysticercosis complex and our important monitoring source of the diseaseincidenceon animals. Theimpact of cysticercosisoccurrencehas been linked to economiclossesassociated to food production, meaning losses of US $\$ 420,000,000$ per year in South A merica (OPAS, 1994). In Brazil, despitethebackground of many states with high incidence of thedisease, adetailed study of thiszoonosisall over thecountry hasnotoccurred until thepresent. In bovinecattle, cisticercosishasbeen themostdiagnosed parasitic zoonosis in slaughter houses-cold stores and the main reason for meat condemnations and conditional carcass utilizations. The present study was aimed to determine the prevalence of cysticercosisin animal carcassesfromslaughter houses under federal surveillancein Rio deJaneiro State, from 1999 to 2003.

KEY WORDS: Bovinecisticercosis, Rio deJanei ro, slaughter houses-cold stores.

\section{INTRODUÇÃO}

O Estado do Rio de Janeiro não apresenta uma economia tradicional voltada para a pecuária, principalmenteadecorte, masseu rebanho vemapresentando um aumento consi derável, nesta última década. A escolhaderaças bovinas melhoradas geneticamente, voltadas para o ganho de peso mais rápido e com vistas às necessidades do mercado externo, destaca a qualidade da pecuária deste Estado.

O Brasil possui situação privilegiada no cenário dabovinocultura, apresentando-secomo detentor do maior rebanhocomercial do mundo, possuindotodas as condições para o setor das indústrias de carne e derivados al cançar uma maior partici pação no mercado internacional (ALVES, 2001).

É desuma importância o desenvolvimento deum programa de sanidade animal, para o controle de enfermidades que causam perda de produção e produtividade à pecuária nacional e oferecem riscos à saúde do homem (LYRA \& SILVA, 2002).

A cisticercose é uma enfermidade parasitária provocada pela ingestão de ovos de Taenia sp., os quaisapósseremingeridospelosbovinos, irãodesenvolver no organismo do animal o Cysticercus sp., vulgarmentedenominado cisto, o homemao ingerir a carne bovina, mal cozida, pode-se infecta.

A prevenção da teníase humana apóia-se em um conjunto demedidas, quevisam impedir a infecção do homem pelaT aeniasp., bloqueandoo ciclodetransmissão deste parasita. Entre essas medidas, a inspeção sanitáriadecarnesreal izadaem matadouros-frigoríficos, representa um importante método preventivo, impedindo que carcaças imprópias para consumo humano sejam comercializadas (CorRêA etal., 1997). O matadourovem sendo utilizado desdesua introdução em Roma, por Galeno, até os dias atuais, como um instrumento deprofilaxia das doenças transmissíveis e de vigilância epidemiológica (FukUDA et al ., 2003).

A cisticercose bovina passa imperceptível aos ol hos do criador edo veterinário, quesó dá conta da importância do controledesta enfermidade, quando do momento do abate, poisduranteavidado animal, esta nunca Ihe causou qualquer sinal ou sintoma que justificasse tratamento medicamentoso, medidas preventivas ou profiláticas. As carcaças ou órgãos parasitados com oCisticer cus bovis (GoeZE, 1782) podem ter destinos variados, dependendo do grau de acometimento, seguindo para a salga, conserva, congelamento atéa condenação total , causand ograves prejuízos a quem cria, recria e/ ou engorda o gad o para abate. O preço da carcaça estaria na faixa de $R \$ 850,00$ a $R \$ 1.000,00$ eos prejuízos são estimadosem casos decisticercoseviva numa queda deaté $30 \%$ no preço do bovino abatido, éo querel ataGuIRRA (2002).

Outro problema importanteé a clandestinidade do abate, que atinge el evados percentuais, como as próprias autoridades federais o declaram, acarretando sério problema à saúde pública egravíssimos danos à indústria idônea eorganizada. Valeressaltar, que as principais causas do abate clandestino estão relacionadas desde a fal ta defiscal ização (número reduzido deprofissionais), punição rígidaaos infratores, a sonegação de taxas e impostos, baixo custo operacional ereduzi do investimento eminstalações, facilidadedecol ocação do produto no mercado varejista local, desinformação do consumidor, além do poder sócio-enotificação compulsória da doença (Fundação N ACIONAL dA SAÚdE, 1996). Exceto em Ribeirão Preto, São Paulo, o coeficiente de prevalência, baseado na notificação compulsória, é de 67 casos/ 100.000 habitantes (Ribeirão Preto, 1998).

O complexoteníase-cisticercosedeterminadopela Taenia saginata (GoezE, 1782) apresenta distribuição cosmopolita, estandoamplamentedifundidonamaioria dos países em quehá criação bovina. O conhecimento da prevalência da doença, tanto no homem quanto nos animais, é deficiente devido à falta de dadossistemáticos, fidedignosecomparáveis(SouLSBY, 1975; PAWLOWSKI, 1982). 
A prevalência da cisticercose bovina provém de dados dos serviços de inspeção veterinária em matadouros. A inspeção de carnes é realizada em vários países do mundo, porém os métodos de diagnóstico post-mortem utilizados geral mente possuem diferenças, comtaxas deprevalênciavariáveisnas diferentes áreas geográficas do mundo, de acordo com fatores sociais e culturais (OPAS, 1994).

A Divisão de Inspeção de Carnes de Brasília (DICAR), recomendou através da circular denúmero 054/ 88, de 19 de maio de 1988, a implantação do método de exame do diafragma, criado por SANTOS (1984), como normarotineiranainspeçãopost-mortem, em todos os estabelecimentos de abate do país sob Inspeção Federal, fato esteque atéo momento, ainda não se concretizou (FukUdA et al ., 2003).

No Estado de São Paulo, a prevalência de cisticercose bovina foi detalhadamente pesquisada por UNGAR (1992), que verificou que de um total de 896.654 de animais abatidos no ano de 1986, 48.957 foramregistradoscomcisticercose, oquecorrespondeu a uma prevalência de $5,46 \%$.

O objetivo destetrabal ho foi fazer umlevantamento dacisticercoseem carcaças bovinas do Estado do Rio dejaneiro, abatidasem matadouros-frigoríficos, submetidosao Serviço del nspeçãoFederal, no período de 1999 a 2003 (BRASIL, 2004), edetectar a prevalência no referido Estado.

\section{MATERIAL E MÉTODOS}

A inspeção do post-mortem realizada pelo SIF forneceinúmeros dados, todos registradosem mapas de abate diário, semanal e relatórios mensais e anuais, quesão preenchidos pelo médico veterinário responsável eenviados ao Ministério da Agricultura.

Neste trabalho utilizou-se dados referentes aos abates de 494.620 bovinos, real izados no período de 1997 a 2003, originários de 38 M unicípios do Estado do Rio de Janeiro: Araruama, Bom Jardim, BomJesus de Itabapoana, Cabo Frio, Cachoeiras de Macacu, Cambuci, Campos dos Goytacazes, Cantagalo, Cardoso Moreira, Carmo, Casimiro de A breu, Cordeiro, Duas Barras, Guapimirim, Itaboraí, Itaguaí, Italva, Itaocara, Itaperuna, Lajedo Muriaé, M acaé, Macuco, Miracema, N atividade, Porciúncula, Quissamã, Rio Bonito, Rio das Ostras, Santa M aria Madalena, Santo Antônio de Pádua, São Fidelis, São Francisco de Itabapoana, São J osé de Ubá, São Sebastião do Alto, Saquarema, Silva Jardim, Sumidouro eTrês Rios. A rastreabilidade foi realizada com as análises das Guias de Trânsito de A nimais (GTAs), pertencentes aos arquivos de órgãos responsáveis, visando o conhecer a procedência do rebanho eo mapeamento das regiões endêmicas.
A inspeção decarnes, realizadaem matadourosfrigoríficos, possibilitou o diagnóstico dacisticercose bovina, através do exame post-mortem. N esteexame, foram realizadas incisões na musculatura esquelética e em órgãos onde os cistos são encontrados com mai or freqüência e o diagnóstico se fez através da sua visual ização macroscópica. N o presente trabal ho, foi computado o total de ocorrência de cistos, sem especificar a sua localização. A cisticercose pode ser classificada como viva ou calcificada/ mineralizada.

A análise estatística foi realizada com vistas ao cálculo da prevalência e possíveis associações entre avariável dependentecisticercoseeas variáveisindependentes sob estudo.

\section{RESULTADOS}

Numtotal de494.620animaisabatidospertencentesa 38municípios do Estado do Rio deJaneiro, 9.656 (1,95\%) apresentaram o parasitismo por Cisticercus sp., possuindo cisticercoseviva(0,8a 1\%)ecal cificada (99,0\%), no período de 1997 a 2003 (Tabela 1). O município queapresentou o maior índiceno período estudado, foi o município de Duas Barras com uma média total de 118 animais abatidos/ ano e uma média de $4,29 \%$ de acometimento por cisticercose. A contagem no matadouro foi feita da seguinte forma: carcaça, cabeça, língua, coração, diafragmaeesôfago, estes são os chamados "sítios de predileção".

Tabela 1- Prevalência decisticercose bovina em animais abatidosde38municípios do Estado do Rio dejaneiro, sob o controle do SIF, no período de 1997 a 2003.

\begin{tabular}{lcrr}
\hline Ano & Animaisabatidos & \multicolumn{2}{c}{ Cisticercose } \\
\cline { 3 - 4 } & & No de casos & $\%$ \\
\hline 1997 & 61.337 & 972 & 1,58 \\
1998 & 95.100 & 1.277 & 1,34 \\
1999 & 103.980 & 1.662 & 1,59 \\
2000 & 69.412 & 1.842 & 2,65 \\
2001 & 81.399 & 1.911 & 2,34 \\
2002 & 41.334 & 1.110 & 2,68 \\
2003 & 42.058 & 882 & 2,09 \\
\hline Total & 494.620 & 9.656 & 1,95 \\
\hline
\end{tabular}

\section{DISCUSSÃO}

Com referência aos resultados da prevalência de cisticercose bovina obtidos no presente levantamento, torna-sedifícil estabel ecer comparaçõescomresultados deoutros trabal hos, devido ao fato denão seter 
encontrado publicações que contivessem a distribuição espacial etemporal da doença no Estado do Rio deJaneiro.

O M unicípio de Duas Barras apresentou o maior percentual $(4,29 \%)$ de animais abatidos com cisticercosenoano de2003, possivel mente, relacionadasàscondições precárias, ou mesmo inexistentes de saneamento básico local.

A ocorrência decisticercosepodeser interpretada como duplo indicador: da situação sanitária deste tipo de exploração e da ocorrência de cisticercose humana, pois os animais se infectam com ovos de Taenia sp. originários defezes do homem, seu hospedeiro definitivo.

Considerando os dados da Tabela 1 , a média total foi de 1,95\%, nos anos de 2000 a 2002 a média da porcentagem deanimais foi de 2,5\%, a faixa aceitável paraum paísem desenvol vimento giraemtorno de $1 \%$ até3\% equando estafaixaéultrapassada, serianecessáriotomar medidas preventivasurgentesparacontrolar tal fato, mas nem sempre isto ocorre (FAO, 1986).

O comércio bovino apresenta as seguintes características: animais podem ser criados em uma propriedade engordados na mesma ou nascerem em uma propriedade e logo após os primeiros meses de vida são vendidos, indo para outra propriedade para a engorda e esta segunda podeser em outro município ou até mesmo em um segundo estado. $O$ animal poderiater seinfectadoemqual quer umadessasfases de sua vida. Seria necessário estudos detalhados, rastreando a origem do problema.

O abate clandestino é uma realidade em vários países, inclui o combate a esteprocedimento entreas medidas prioritárias de um programa decontroledo Complexo Teníase-Cisticercose. Esse tipo de abate é umproblemadegranderelevânciaetemcomo umdos principais motivos a estrutura tributária do país, e enquanto não houver uma reforma tributária o problema persistirá, éo que afirmou MülLER (1997), PresidentedaFederação deAgriculturado Estado do Rio Grande do Sul, em entrevista a RNC.

Segundo os dados da RNC (1993-1996) a prevalência decisticercose poderiaser maior, secontássemos com os dados originários do abateclandestino (MIRANDA, 2002).

Defrentecom estefato, propõe-sea hipóteseneste trabalho que, se $50 \%$ (247.310) do total do abate, ocorridonesteperíodo, fosseonúmerocorrespondente ao abate clandestino, teríamos que acrescentar ao total, e o resultado da prevalência seria de $2,92 \%$ carcaças bovinas parasitadas pelo Cisticercus sp. no período de1997a2003noRiodejaneiro. Obviamente o efetivo de bovinos abatidos desta forma não écomputado nos levantamentos estatísticos oficiais, fato esteque podeatéa conduzir resultados contraditórios que não espel ham a realidade.
Osprejuízosadvindosdacisticercosebovina, como já foi citado, ocorrem na fase final da exploração de corte, ou seja, após o abate, real izado em matadourofrigorífico sob fiscalização estadual ou federal.

A Legislação Brasileira (BRAsIL, 1980) prevêbasicamente 3 destinos: liberação, condenação eaproveitamento condicional, através do frio, salga e pelo calor, sendo que os maiores prejuízos econômicos ocorrem nos dois últimos tipos de destinações. De uma maneira geral, a condenação total é indicada para casos de infestações generalizadas. A liberação da carcaçain natura éprevistaquando for encontrado um único cisto calcificado, após a sua excisão. Nos casos de infecção moderada ou local izada, as carcaças e órgãos afetados podem ser aproveitados, após serem submetidos a um dos seguintes tratamentos: pelo frio (-10 $0^{\circ} \mathrm{C}$ por $10-14$ dias), pelo cal or (àtemperatura mínima de $60^{\circ} \mathrm{C}$ ) e pela sal ga (à temperatura de $10^{\circ} \mathrm{C}$ ) (Ornanizacion Mundial de la Salud, 1979).

No Brasil, é difícil quantificar, com precisão, os valores decorrentes destas perdas, devido ao fato das publicações a este respeito serem esporádicas e não atual izadas, ainda são pou cos os estados na federaçãoqueconhecemataxadeprevalênciadecisticercose bovina em seu rebanho. Aliando-se ao fato de que a cisticercose bovina não éexclusivamente um problema de ordem econômica, mas também constitui um sério risco para a saúde pública.

M uitos casos positivos, mesmo no serviço deinspeção, podem passar despercebidos, principalmente onde a infecção é moderada. Há também restrições, em termos comerciais, pois não é permitida incisões emcarnesnobres, isto depreciariaseu valor demercado, mas não excluiria a possibilidadedeseencontrarem infectadas, expondo o consumidor ao risco.

Outros fatores, apesar deserem decaráter genérico, podem limitar a eficiência diagnóstica da cisticercosebovina. São representados pelas características do estabelecimento, como por exemplo, a má iluminação e o excesso de trabal ho decorrente deum grandenúmero deanimais abatidos, a pouca experiência do pessoal envolvido podendo, até, resultar em diferentesíndices dedetecção daenfermidadedentro de um mesmo estabel ecimento.

O SIF funciona não somente como um órgão de inspeção, mas também como um centro detentor de dados, sobre patologias de grande importância na Saúde Pública, sua contribuição epidemiológica é fundamental, mas por não processar devidamente, estes dadosinexploradospodem seperder, enquanto poderiam ser utilizados em mapeamentos epidemiológicos estaduais das enfermidades mais freqüentes, auxiliando a elaboração de programas decontroleeprofilaxiadezoonoses maisfreqüentes no país, associando-se a outros órgãos de saúde municipais, estaduais ou federais. É papel do médi- 
co-veterinário inspetor interagir neste processo e reverter estasituação, tornando maisdinâmicaasua atuação.

REFERÊNCIAS

Alves, D.A. As dificuldades na inspeção de frigoríficos brasileirosnomercado internacional:Umestudo sobreacomercialização dacarnebovinain natura. Revista N acional da Carne, v.25, n.291, p.96-114, 2001.

BRASIL. Ministério daA gricultura. Regulamentodeinspeção industrial esanitáriadeprodutos deorigem animal.Brasília: Ministério daA gricultura, 1980.116p.

BRASIL. Ministério da Agricultura. Serviço de Inspeção Federal - SIF. Relação de abate de doença por procedênciaemunicípio. Disponível em: $<$ http:/ / extranet. agricultura.gov.br>. A cesso em: 15 set. 2004.

Center for Disease Control. Recommendations of the International Task Force for Disease. M orbidity and M ortality W eekly Reports, v.42, p.1-25, 1993.

Corrêa, G.L.B.;A da m S, N A.;A nGNES,F.A.;Grigoletto ,D.S. Prevalência decisticercose em bovinos abatidos em Santo A ntônio das Missões, RS, Brasil. R evista da Faculdade de Z ootecnia, Veterinária e A gronomia, v.4, n.1, p.43-45, 1997.

FAO. A nimal health yearbook 1986. Rome: FAO, 1986. 51p. (A nimal Production and Health Series, 26).

FERTIG ,D.L.\&DORN, C.R.Taenia saginatacysticercosisin an Ohio cattlefeeding operation.J ournal of theA merican Veterinary Medical Association, v.186, p.1281-1285, 1985.

FukUdA,R.T.;SANTOS, I.F.; A ndRADE, C.R. Estudo comparativo entre técnicas de inspeção do diafragma para o diagnóstico da cisticercose bovina. Disponível em: «tttp:/ / www.bichoonline.com.br/ artigos/ ha0003. htm>. A cesso em: 6 abr. 2003.

Funda ÇÃo Nacionalda SAúde (Brasil). Projetoparaocontrole do complexo teníase/cisticercose no Brasil. Brasília: FUNASA, 1996. 53p.

Germ An o, P.M .L. Zoonosesesaúdepública. HigieneA limentar, v.1, n.2, p.73-79, 1982.

GuirRA, F.Cisticercoseaindaprovocaquedade $30 \%$ no preço doboi. Disponível em: <http:/ / www.revistasafra.com. $\mathrm{br} / 2002-03 /$ cisticercose ainda provoca.htm $>$. Acesso em: 6 abr. 2002.

IBGE. A nuário estatístico do Brasil - 1987/1988. Rio deJaneiro: Fundação InstitutoBrasileirodeGeografiaeEstatística, 1988. v.48.
LYRA, T.M.P. \& SLVA, J.A. O componente social e sua importância naplanificação em saú deanimal. Revista CFM V , v.8, n.26, p.11-20, 2002.

MIRANDA, Z.B. Inspeção de produtos de origem animal. Revista CFM V , v.8n.26, p.21-26, 2002.

MüLLER,G. A ganânciatributáriafavoreceoabateclandestino. Revista N acional daC arne, v.21, n.240, p.6-10, 1997.

Organización Mundial de LA Salud. Zoonosis parasitárias: informe de un Comité de Expertos de la OMS, con la participación de la FA 0. Ginebra: OPS, 1979. 135p.

Organización Panamericana de la Salud. Epidemiologiay control da la teniasis/cisticercosis en A merica Latina. $\mathrm{N}$ ew York: OPS, 1994. 1CD.

PawLowskı, Z. Taeniasis and cysticercosis. In:JA COBS, L.\& Arambulo, P. (Eds.).Parasiticzoonosis. BocaRaton:CRC Press, 1982. p.313-348.

ReY, L. Bases da parasitologia médica. Rio de Janeiro: Ed. Guanabara Koogan, 2002. 379p.

RodRIGUES, R.R. Acabar comamatança clandestinaémeta prioritária. Revista N acional da Carne, v.18, n.201, p.35, 1993.

SANTOS, I.F. D iagnóstico da cisticercosebovina em matadouros: novas técnicas de exame de esôfagos e diafragmas. 1984. 127p. Tese(Doutorado) - FaculdadedeSaúdePública, UniversidadedeSão Paulo, São Paulo, 1984.

Ribeirão Preto (SP). Informativo epidemiológico de Ribeirão Preto. Ribeirão Preto: SM SRP, 1998. v.2., n.2.

SILVA, L.J. A ocupação doespaço eaocorrênciadeendemias. In: Barata, R.B. \& Briceño-León, R. (Eds.) D oenças endêmicas: abordagens sociais, culturaisecomportamentais. Rio de Janeiro: Editora FIOCRUZ, 2000. p.139-150.

SouLSBY, E.J.L. Teniasisy cysticercosis: el problema en el viejo mundo. In: REUNION INTERAMERICANA SOBRE EL CONTROL DE LA FIEBRE AFTOSA Y OTRASZOON OSIS, 7., 1975, Puerto España. Resumos. Puerto España: Organización Panamericana de la Salud, 1975. p.136-142.

Takayanagui,O.M.;Castro eSilva,A.A.C.;Santiago, R.C.; Odashima, N.S.; Terra, V.C.; Takayanagui, A.M.M. N otificação compulsória da cisticercoseem Ribei rão Preto-SP. A rquivo Neuropsiquiatria, v.54. p.557-564, 1996.

UNGAR, M.L. Prevalênciadacisticercosebovina no estado deSão Paulo (Brasil).R evista deSaúdePública, v.26,n.3, p.1-12, 1992.

Recebido em 24/ 7/ 05

Aceito em 31/ 3/ 06 